\title{
What to know before starting the process of publishing in a medical journal
}

\author{
Marco Masina, ${ }^{1}$ Paola Gnerre, ${ }^{2}$ Paola Granata, ${ }^{3}$ Roberto Nardi ${ }^{4}$ \\ ${ }^{1}$ Stroke Care Area - Geriatric Ward, Bentivoglio Hospital, Bentivoglio (BO); ${ }^{2}$ Internal Medicine, San Paolo Hospital, Savona; \\ ${ }^{3}$ PAGEPress Scientific Publications, Pavia; ${ }^{4}$ Internal Medicine, Bologna, Italy
}

\begin{abstract}
The Authors analyze the path of the publication of a study starting from the choice of the journal. Subsequently they summarize the different systems of data reporting and how to format a manuscript according to well-established international editorial structures. In conclusion they suggest how to positively manage a rejection.
\end{abstract}

\section{Introduction}

Getting a work published in a medical journal has become a divide among doctors. For researchers the motto publish or perish clearly describes a condition in which the loop is no publication, no chance to publish in the future. For clinicians who work in hospitals publishing is not mandatory but it helps boosting a personal career. At least, publishing lets others know what is going on in your ward or hospital. In this paper we analyze the main problems a clinician can face in order to publish. The Authors start from the choice of the journal to which the manuscript is sent, then describe how to report data according to different types of study. They summarize how to write a paper according to well-established editorial frameworks. They conclude by describing what to expect from the peer review process and how to positively manage a rejection.

Correspondence: Marco Masina, Stroke Care Area - Geriatric Ward, Bentivoglio Hospital, via Marconi 35, 40010 Bentivoglio (BO), Italy.

Tel.: +39.051.6644490 - Fax: +39.051.6644491.

E-mail:marco.masina@ausl.bologna.it

Key words: Publication process; writing a scientific manuscript; choosing the best journal.

Conflict of interest: the authors declare no potential conflict of interest.

Received for publication: 27 January 2017.

Revision received: 26 May 2017.

Accepted for publication: 8 June 2017.

This work is licensed under a Creative Commons Attribution NonCommercial 4.0 License (CC BY-NC 4.0).

CCopyright M. Masina et al., 2017

Licensee PAGEPress, Italy

Italian Journal of Medicine 2017; 11:325-330

doi:10.4081/itjm.2017.842

\section{The journals}

In the last 20 years competition among scientific and medical journals has been fierce. Some journals raised to incredible scores of impact factor (IF). ${ }^{1}$ They achieved that goal by becoming more selective and reducing the acceptance rate to a low $5-10 \%$. In this process reviewers often reject innovative papers and prefer studies proposing small innovation on well-established results. Top journals often ask an authoritative scientist to review the literature on a specific topic in order to boost citations and consequently increase their impact factor. Doubts have been risen on the blind process of peer-reviewing in and of itself or on Big Pharma backing up some studies. For a manuscript coming from a newbie the chances of being published in prestigious journals are really low. The process that leads to acceptance may be a long one. A smart move is to join group of researchers backed up by an established author, then to climb up the ladder, and finally to have the name as first in a work. That works better for academics than for clinicians.

Clinicians have large experience on routine clinical care. Is the description of their daily experience interesting for a top-ranked journal? Frankly speaking, usually not. Exceptions exist, but they are just that: exceptions.

In any case, if a study has been correctly planned and carried out, there are some results that can be presented. Instead of aiming high, it is better to look for a journal to be read by your peers. The chances of being published increase if your results are of interest to the scholarly public of the journal. So the choice of the journal to which the paper is submitted is critical. Methods of measuring the quality of Scientific Journals are reported in Table 1. ${ }^{1-17}$

\section{How to report data according to the type of study}

In this paper we only face the problem of publishing a manuscript without taking into account how to 
Table 1. How to measure quality of Scientific Journals: institutions and tools.

\section{Institute for Scientific Information standardized measure}

The Institute for Scientific Information (ISI), currently known as Thomson ISI, was founded by Eugene Garfield in 1960 and subsequently acquired by Thomson Reuters, with the aim of offering bibliographic database services. In particular, ISI offers citation indexing and analysis by covering thousands of academic journals. In a paper published in Science in 1955, Garfield first described his ideas about possible applications of automatic methods on scientific documentation, which are widely used today. Referring to scientific articles, Garfield wrote that this would be particularly useful in historical research when one is trying to evaluate the significance of a particular work and its impact on the literature and thinking of the period. Such an 'impact factor' may be much more indicative than an absolute count of the number of scientist's publications. ${ }^{2}$ Publications in print are gradually giving way to electronic publications: database such as the Web of Science are widely used and offered by ISI to online network users. It is adopted by national higher education consortia in 14 countries. It contains details of over 23 million source articles containing 300 million cited references extracted from science, social science, and arts and humanities journals covered in ISI's services. ${ }^{3}$ Accordingly, ISI has developed a number of products.

\section{The scientific citation index}

The scientific citation index (SCI) database represents the ISI most valuable asset because its content can be processed in many ways to give new products. ${ }^{3}$

The SCI database can be defined as a comprehensive citation index containing all references of a source article which appear in bibliography or footnotes in a large number of multidisciplinary and specialties journals. Using a citation index, the reader has the opportunity to compare and understand differences among papers by confronting the evolution of a concept in time. Therefore, it answers the question of What has happened since? As a sophisticated searching tool, the citation index is concept-oriented and needs a wide knowledge of the starting point. A secondary application of the SCI is that of a conventional index to list publications from any author. ${ }^{4}$

\section{The impact factor}

A journal's impact factor (IF) is a ratio between the numerator, represented by the number of citations in the current year to any items published in a journal in the previous 2 years, and the denominator, represented as the number of citable items (articles, reviews, proceedings, or notes, but not editorials or letters to the editor) published in the same 2 years. ${ }^{5}$ It was originally conceived as a tool for librarians and publishers to provide information about the citation performance of a journal as a whole. Over the last few decades IF has expanded to assess the quality of specific articles and the research performance of individual investigators. ${ }^{6}$ Actually Eugene Garfield, the IF inventor and principal shareholder of the Institute for Scientific Information, affirmed that IF should not be used in evaluating scientific research. ${ }^{7}$ Garfield wrote: The source of much anxiety about journal impact factors comes from their misuse in evaluating individuals, e.g., during the habilitation process ${ }^{8}$ That is because the impact factor is not an absolute measure of the quality of a journal, but of its influence, ${ }^{9}$ and it is not a validated tool in the assessment of the professional skills of hospital doctors. ${ }^{10}$ Nevertheless it is widely credited in the academic world in benchmarking universities or in scholars competition. Researchers should not be judged according to the impact factor of the journal in which they publish their contributions, but on the basis of scientific accuracy, quality of studies, dissemination and reproducibility of results. ${ }^{11}$ Review articles typically collect more citations than research articles, and journals often publish reviews in order to raise their impact factor. ${ }^{12}$

Which other parameters, besides the IF, should be considered for judging the quality of a journal? In the goal of attracting high-quality papers, journals should shift their attention away from IF, focusing on other issues, such as ensuring a fast, fair and professional review process, increasing online availability, decreasing publication costs, and improving post-acceptance production assistance.

Major disadvantages of using an impact factor as the sole method of assessment ${ }^{5}$

- Journal impact factors are not statistically representative of individual journal articles

- Journal impact factors correlate poorly with actual citations of individual articles

- Review articles are heavily cited and inflate the impact factor of journals

- Long articles collect many citations and give high journal impact factors

- Short publication lag allows many short-term journal self-citations and gives a high journal impact factor

- Selective journal self-citation: articles tend to preferentially cite other articles in the same journal

- Books are not included in the database as a source for citations

- Database has an English language bias

- Database is dominated by American publications

- Impact factor is a function of the number of references per article in the research field

- Research fields with literature that rapidly becomes obsolete are favored

- Impact factor depends on dynamics (expansion or contraction) of the research field

- Small research fields tend to lack journals with high impact

- Citation rate of an article determines the journal impact, but not vice versa

- Availability of research material to scientists and researchers worldwide determine their pattern of citation i.e., some references are not available for many scientists worldwide

- Language knowledge affects the number of articles cited for a publication

\section{The Hirsch factor}

The Hirsch index (H-index) is a tool to evaluate the scientific performance of authors. The H-index of an author is $\mathrm{N}$ when at least $\mathrm{N}$ papers have been cited $\mathrm{N}$ times. The H-index has an intrinsic uncertainty, which depends on scientific sector, the age of the scientist and the number of its papers. An H-index can only increase, even though an author has become inactive and the H-index of a young excellent scientist is always small. ${ }^{13,14}$ To improve or complement the $h$-index, many $h$-type indices have been proposed. ${ }^{15}$

\section{The Open Access journals}

A final consideration is about Open Access Journals (OAJ). The Internet has changed the approach to scientific and scholarly literature, giving way to an Open Access web-based literature, that is (or should be) digital, online, free of charge, free of most copyright and licensing restrictions, and entirely compatible with the peer review process. The challenge for OAJ is to spread the knowledge allowing freely availability to contents. That goal is usually achieved by suppressing/reducing charges to readers and/or to authors and institutions. Often charges are fully waived for authors of economically disadvantaged countries.

Digital publishing is less expensive than printed literature but the process cannot become costless and someone has to pay the costs. Recently, The Guardian reported that the mean cost of publishing an article in PLoS One (a leading Open Access Journal) is about £850, far lower than the $£ 6700$ claimed by Elsevier publisher. ${ }^{16}$ In OAJ editors and peer-reviewers are not paid. OAJs use auxiliary services to pay publishing costs, such as earnings from other publications, from advertising, or from priced add-ons. Many organizations like Health Care Trusts or Scientific societies pay on behalf of their affiliated allowing them to have their work published for free.

OAJ archives or repositories (property of institutions, such as universities and laboratories) are also involved in supporting authors in archiving their preprints without asking Editors' permission. The majority of journals already permit authors to archive their postprints. ${ }^{17}$

In Italy, the CRUI (Conferenza dei Rettori delle Università Italiane - Conference of Italian University Deans) recognized the role of OAJ publishing in spreading scientific researches and promoted several conferences, documents and projects to make authors and readers aware and to promote publishing in OAJ journals (https://www.crui.it/open-access.html). 
plan and carry out a study. That is a complex task and specific books describe the necessary steps. In preparing your study remember to answer the questions: What kind of study is it? Is it a clinical trial or a case report? Is it a prospective or a retrospective one? Where does it stand in the hierarchy of studies?

A clinical trial is a tough task. A clinical trial is a study that prospectively assigns human subjects to intervention, concurrent comparison, or control groups to assess the cause-and-effect relationship between medical interventions and the health outcome. Medical interventions include drugs, surgical procedures, devices, behavioral treatments, process-of-care changes, etc. Owing to their high cost, those studies are usually sponsored by a Pharma company. It must be remembered that almost all the journals require a clinical trial to be formally registered.

In a prospective study the sample is chosen on exposure, it is followed over time and the focus is on outcome (e.g., cohort study). A prospective study requires to be planned in advance. A statistical model must be targeted to show significant results and hopefully confirmed. The study must be approved by the ethical committee and patients' informed consent must be collected. Comparison between different therapeutic strategies sometime needs a number of patients larger than that provided by a single hospital. A multi-center prospective study is far more expensive and time consuming and can only be carried out by a scientific society or a Regional or Local Health Care Authority.

In a retrospective study the sample is chosen based on outcome, looking back for the presence or absence of exposure. A retrospective study seems easier to perform, but data handling may still be challenging. A retrospective real life study requires a coherent collection of both administrative and clinical data that sometime do not match because of coding inaccuracy. Accessing official databases requires a formal consent. Missing data or lost patients may endanger the analysis and must be accounted for. ${ }^{18}$

When the study is completed and data are ready to be reported on a paper, authors have to consider that their manuscript must fulfill some criteria. There are many guidelines available on reporting results of clinical trials, observational studies or meta-analyses.

The CONSORT (CONsolidted Standards of Reporting Trials) is a guideline containing a checklist of 25 items that correspond to the contents of each part of the paper (Title, Abstract, Introduction, Methods, Results, Discussion and Other information). ${ }^{19}$ The STARD (STAndards for the Reporting of Diagnostic accuracy studies) is a guideline to check the diagnostic accuracy of what authors state in the paper. ${ }^{20}$ The STARD flow diagram is widely present in the section reporting methods of a large number of clinical studies.

The STROBE (Strengthening the Reporting of
OBservational studies in Epidemiology) guideline contains recommendations to the three main analytical designs used in Observational researches: cohort, case-control and cross-sectional studies. The STROBE Statement is being endorsed by a growing number of biomedical journals. ${ }^{21}$

The PRISMA (Preferred Reporting Items for Systematic Reviews and Meta-Analyses) represents an evidence-based set of items for reporting systematic reviews and meta-analyses. ${ }^{22}$

\section{How to write a paper - The IMRaD structure}

Any submitted paper must have a Title and be divided into sections. The InternationaI Committee of Medical Journal Editors (also known as the Vancouver Group) published a guideline that requires authors to format their manuscripts according to the IMRaD structure (Introduction, Methods, Results and Discussion). This has obtained the absolute leadership since the eighties. ${ }^{23}$ More recently the second section has been named Materials and Methods and a brief Conclusion may be added at the end of the paper.

Presently the components of most manuscripts are: Title, Introduction, Materials and Methods (including Statistical analysis), Results, Discussion, Conclusions and References. Those parts must be complete and coherent.

Title: according to the Publication Manual of the American Psychological Association, a title should summarize the main idea of the manuscript in a simple way and when possible with style..$^{24}$

That means that a title should be short - often journals limit the number of words - but at the same time it has to summarize the research and when possible the results. Moreover the title has to take into account the journal targets and to appeal to the scholar community which will read it.

In writing the title always check for the editorial formatting rules. Add a subtitle only if requested by them. Remember that American journals often require capital letters on each noun (but not preposition) while British journals do not.

The title can help the work get visibility. If the title contains references to the main topic the study can be found more easily by search engines and the chance of being cited widens. Of course, the same criteria have to be considered in choosing appropriate keywords.

Introduction: it briefly reviews the present knowledge and the unmet needs of the fields and explains the aim of the study. Long introductions are boring and can distract the reader away from the point. Usually a good introduction does not exceed 3 paragraphs. The last paragraph briefly summarizes the aim of the work.

Materials and methods: they are not difficult to report. In this section authors describe the experimental 
setting, the inclusion and exclusion criteria, the final number of patients considered, and the outcomes. Experimental animal studies are rarely performed by clinicians. Large ethical issues about animal testing have been summarized by the 3 Rs (Replacement, Reduction and Refinement) since the late fifties. Researcher should prefer non animal studies when possible, the number of animals should be reduced to the minimum, and their pain shoud be minimized or alleviated. In Europe, since January 2013 Authors should declare that the experiment was carried out according to the Directive 2010/63/EU about the protection of animals used for scientific purposes.

Lab studies may be included in clinical reasearch, expecially in some fields as microbiology and pathology but they can also concern psychology. In order to eliminate the effect of external viariables, lab studies take place in a controlled environment, which has to be accurately described in this section. Before publishing exceptional results, it must be remembered that results should be replicable in other laboratories.

The statistical analysis has to be described in a specific paragraph together with the demographic, clini$\mathrm{cal}$, and outcome variables. The statistical program has to be cited.

Results: clearly reporting results in good English is a difficult task for native speakers too. Consider that reviewers (and readers) can only learn about your experience by reading the paper. The language has to be clear. Data are better reported in tables and described in the text following the order of presentation. Precision and consistency are essential in reporting results, so that to avoid uncertainties. The vocabulary used in a scholarly paper should be chosen carefully. Repetition of some critical words may be preferable to the use of synonyms. Short sentences are the best choice.

Discussion: in this part results are compared to those of the literature. It is the longest part of the paper and it is expected to contain about 6-7 paragraph. You do not have to follow the same order as in Results, since you follow a logic process. Nevertheless the presentation of topics must be clear, concise, and logical. A starting paragraph shortly reporting the current literature is usually followed by a second paragraph summarizing main results. Differences must be emphasized confirming the importance of the results. The conclusions should be supported by the data. Limits in the project or in the results must be appropriately reported and discussed.

Conclusions: despite conclusions are not included in the IMRaD format, almost all manuscripts end with one summarizing the main results of the study in no more than 2-3 paragraphs.

Declaration, plagiarism and disclosure: almost all journals require a declaration that the submission neither has been previously published, nor it is under con- sideration to another journal for publication. A double or triple submission at the same time may seem smart but is hard to handle and it is not ethically correct. Remember that some reviewers collaborate with more than one journal.

Plagiarism is an important issue. Never forget that tools exist, which can easily discover plagiarism and that all publishers check your paper on a specific database.

At the end of the paper a full disclosure of each author's interest should be reported. Grant and financial support must be honestly declared.

Other extremely important aspects of papers that may help or hamper the chance for a paper to be published are the Letter of presentation, the Abstract, the English language, and the References.

Letter of presentation: its importance is often underestimated. An editor-in-chief receives a large amount of papers and makes up a first selection. In the letter you introduce yourself and your group to $\mathrm{him} / \mathrm{her}$. The Editor has to save his/her reviewers time. Moreover sometimes journals received fake studies deliberately sent in order to check out their selection process. In the letter of presentation you have to briefly describe who you are, where the study was carried out, and if any papers of yours on this topic had been published previously. That does not mean you have to boast your group as a top scorer, but you have to gain the editor's trust. Having the letter written by a trusted author may help to have your paper to pass the first filter.

Abstract: perhaps the abstract is the most critical part of the work. It is the first part of the paper to be read after the presentation letter and often editors never read beyond it. Many studies are discarded because of an unsatisfactory abstract. First, respect the journal rules about format and number of words. So in around 250 words, the abstract represents the only chance to summarize the value of your study, to show the adequate standard of both project and methodology, and to emphasize the relevance of the results. Somebody suggests that it must be written before starting the paper. Surely it cannot be considered as a meaningless and boring part of the job.

English language: English is the ruling language in science and unfortunately we are not native speakers. To this, add the limits of the teaching we were given during school time and the result is very often almost not-understandable and sounds like It-English (Italian-English). Those two languages differ a lot. If you want to write a readable paper you have to think in English, and not simply have your Italian thoughts translated into English. Italian uses long sentences enriched by clauses, subordinates, etc. English people rarely do that! They write short sentences. Take note that native English speakers almost never use semi- 
colons (;) ! So start forgetting them. Of course an accurate spell checking of the paper is mandatory. Typical Italian mistakes are missing ' $s$ ' in plurals and in third person of simple present verbs. Relying on proofreading is a good option but you must be informed if the journal is published in British or American English. There are subtle differences that can make you waste your time and money (e.g., realise $v s$ realize or favour $v s$ favor).

References: references should be prepared strictly according to a definite style, usually Vancouver, Harvard or the American Psychological Association (APA) style. Those styles differ and should not be mixed up. APA Style is seldom used. In this case the references are arranged alphabetically, by the last name of the first author. If the reference list includes two or more entries by the same author, they are listed chronologically with the earliest first. In APA Style the capitalization can follow two different methods: i) title case in which each word in the title of the citation is capitalized, except for articles, prepositions, conjunctions or; ii) sentence case in which only the first word and nouns are capitalized.

Harvard Citation Style or Harvard Referencing System is a misnomer that has no official institutional connection to the famous University. ${ }^{25}$ It reports in the text both author's name and year in parenthesis and refers the reader to the references that are listed in alphabetical order.

Many Harvard style guides edited by different institutions are available online.

Medical journals prefer the Vancouver style. ${ }^{23}$ References must be numbered consecutively in the order in which they are cited in the text (not in alphabetical order as in Harvard or APA styles), and they must be identified in the text by Arabic numerals in superscript after dots and commas.

The reference list must include all references cited in the text.

The capitalization of the title follows the sentence case rule (only initial word and nouns are capitalized). According to the Vancouver Style: author's surname is followed by the initial of the name without dot.

Titles of journals are capitalized too and abbreviated according to the Index Medicus. The list of the Journals Indexed can be found at: http://www.ncbi.nlm.nih.gov/ books/NBK3799/

Where available digital object identifier (DOI) should be included in the reference. DOI is a unique alphanumeric string that is used to identify a certain source (typically journal articles).

URLs for the references should be provided directly within the MS-Word document.

As an example we report that the Italian Journal of Medicine asks authors to write the References section according to the Vancouver style as follows: i) more than three authors, cite 3 authors, et al. If the paper has only 4 authors, cite all authors; ii) title style: sentence case; please use a capital letter only for the first word of the title; iii) journal titles mentioned in the References list should be abbreviated according to the following websites: a. ISI Journal Abbreviations Index (http://library.caltech.edu/reference/abbreviations/); b. Biological Journals and Abbreviations (http://home. ncifcrf.gov/research/bja/); c. Medline List of Journal Titles (ftp://ftp.ncbi.nih.gov/pubmed/J_Medline.txt); iv) put the year after the journal name; v) never put the month and day in the last part of the references; vi) cite only the volume (not the issue in brackets); vii) pages have to be abbreviated, e.g., 351-8.

To ensure the correct citation format, please check your references in the PubMed database (http://www. ncbi.nlm.nih.gov/pubmed).

The ultimate Guidelines for Authors of the Italian Journal of Medicine are available at: http//www. italjmed.org/ijm/about/submissions\#authorGuidelines

\section{Formatting a manuscript}

Italians are a little bit anarchic. They seldom read the rules, and rarely obey to them. In Italy rules can be easily overcome. Unfortunately this is not the case in scholarly journals. The word limits that are set for the abstract and the paper must be respected. Both the abstract and paper must be written according to the structure and formatted in sections as requested. References have to be limited to the number allowed or editors can easily reject the manuscript without reading it.

\section{How to manage a resubmission and a rejection}

If you are invited to resubmit, consider it as a chance to learn from an expert. The best thing to do is to amend your paper taking into account the reviewer's feedback. In case you disagree with the reviewer you should report your point respectfully. You are not expected to quarrel. It is a good idea to write a letter to the editor pointing out the changes you have made.

If your paper is rejected the letter should be read carefully because it often contains questions or comments on the paper that can guide future revisions. When possible, ask politely the editor why the work has been rejected and which changes should be made. ${ }^{26,27}$ That will enhance the chances of being published elsewhere.

A paper could be rejected for a number of reasons: i) it contains no significant contribution; ii) it is boring; iii) it asks too many questions and provides creative answer only to some; iv) it does not fall within the aims and scope of the journal; v) the paper does not conform to the Guidelines for Authors of the journal; vi) key elements in terms of format, word count, number of figures and tables, and references are inac- 
curate; vii) the article contains elements suspected of plagiarism; viii) references are outdated and inaccurate; ix) the language is so poor that the content cannot be assessed by the reviewers; $x$ ) both American or British English words are adopted; xi) Methods are not described in detail; xii) Results are not critically discussed; xiii) the paper is not appropriate: the discussion is illogical and data do not support the conclusions; xiv) Conclusions do not consider current literature.

In case you decide to submit your paper to another journal, completely rewrite the paper to accomplish the other journal instructions and revise the cover letter. Editors laugh when they receive a cover letter addressed to another journal.

In conclusion it is common thought that if you do not publish you perish but if you want to stay in your comfort zone you should not publish at all. ${ }^{28}$

\section{References}

1. Olson L. Guide to academic and scientific publication. How to get your writing published in scholarly journals; 2014. Available from: http://www.proof-reading-service.com/guide/

2. Garfield E. Citation indexes for science. Science 1955; 122:108-11.

3. Cawkell AE, Garfield E. Institute for Scientific Information; Chapter 15. In: Fredriksson EH, ed. A Century of science publishing: a collection of essays. IOS Press; 2001. pp 149-160. Available from: http://garfield.library.upenn.edu/papers/isichapter15centuryofscipub149 -160y2001.pdf Accessed: 11 January, 2017.

4. Garfield E. Science Citation Index. Science Citation Index. 1961, 1, p.v-xvi, 1963. Available from: http:// garfield.library.upenn.edu/papers/80.pdf Accessed: 7 July, 2016.

5. Elsaie ML, Kammer J. Impactitis: the impact factor myth syndrome. Indian J Dermatol 2009;54:83-5.

6. Zupane GKh. Impact beyond the impact factor. Comp Physiol A 2014;200:113-6.

7. Garfield E. How can impact factors be improved?. BMJ 1996;313:411-3.

8. Garfield E. The impact factor and using it correctly. Der Unfall chirurg 1998;48:413.

9. Baethge C. Impact factor - a useful tool, but not for all purposes. Deutsch ArzteblInt 2012;109:267-9.

10. Stefani I, Mazzone A. Certificated clinical competencies are required for Internist career, not only impact factor score. Ital J Med 2015;9:303-4.

11. Al-Shahi Salman R, Beller E, Kagan J, et al. Increasing value and reducing waste in biomedical research regulation and management. Lancet 2014;383:176-85.

12. [No authors listed]. Dissecting our impact factor. Nature Materials 2011;10:644-5.

13. Hirsch JE. An index to quantify an individual's scientific research output. PNAS 2005; 102:16569-72.

14. Hirsch JE. Does the h-index have predictive power?. PNAS 2007;104:19193-8.

15. Zhang C. The $h$ '-Index, Effectively improving the $h$ Index based on the citation distribution. PLoS One 2013;8:e59912.

16 Taylor M. Persistent myth about open access scientific publishing. The Guardian, 17 April 2012. Available from: https://www.theguardian.com/science/blog/2012/apr/17/p ersistent-myths-open-access-scientific-publishing

17. Suber P. A very brief introduction to Open Access; first put online December 29, 2004. Available from: http://legacy.earlham.edu/ peters/fos/brief.htm Accessed: 11 January, 2017.

18. Gnerre P, La Regina M, Ballardini G, et al. How to write a care report? Guidelines for internists. Ital J Med 2014;8:200-3.

19. Shultz KF, Altman DG, Moher D; for the CONSORT Group. CONSORT 2010 Statement: updated guidelines for reporting parallel group randomised trials. BMJ 2010;340:c332.

20. Bossuyt PM, Reitsma JB, Burns DE, et al. STARD 2015: An updated list of essential items for reporting diagnostic accuracy studies. BMJ 2015;351:h5527.

21. Von Elm E, Altman DG, Egger M, et al. The strengthening the reporting of observational studies in epidemiology (STROBE Statement): guidelines for reporting observational studies. J Clin Epidemiol 2008;61:344-9.

22. Moher D, Liberati A, Tetzlaff J, et al. Preferred reporting items for systematic reviews and meta-analyses: the PRISMA statement. PLoS Med 2009;6:e1000097.

23. [No authors listed]. Uniform requirements for manuscripts submitted to biomedical journals. International Committee of Medical Journal Editors. Ann Internal Med 1997;126:36-47.

24. American Psychological Association (APA). Publication manual of the American Psychological Association, 6th ed. Worchester, MA: APA; 2010.

25. Chernin C. The Harvard system; a mistery dispelled. BMJ 1988;297:1062-3.

26. Sullivan GM. What to do when your paper is rejected. J Graduate Med Educ 2015;7:1-3.

27. Bordage G. Reasons reviewers reject and accept manuscripts: the strengths and weaknesses in medical education reports. Acad Med 2001;76:889-96.

28. Colquhoun D. Publish-or-perish: peer review and the corruption of science. The Guardian, 5 September, 2011. Available from: https://www.theguardian.com/science/ 2011/sep/05/publish-perish-peer-review-science 